\title{
On the Union Complexity of Families of Axis-Parallel Rectangles with a Low Packing Number
}

\author{
Chaya Keller* Shakhar Smorodinsky ${ }^{\dagger}$ \\ Department of Mathematics \\ Ben-Gurion University of the Negev \\ Be'er-Sheva, Israel \\ $\{$ kellerc, shakhar\}@math.bgu .ac.il
}

Submitted: Feb 2, 2017; Accepted: Oct 24, 2018; Published: Nov 16, 2018

(C) The authors. Released under the CC BY-ND license (International 4.0).

\begin{abstract}
Let $\mathcal{R}$ be a family of $n$ axis-parallel rectangles with packing number $p-1$, meaning that among any $p$ of the rectangles, there are two with a non-empty intersection. We show that the union complexity of $\mathcal{R}$ is at most $O\left(n+p^{2}\right)$, and that the $(k-1)$-level complexity of $\mathcal{R}$ is at most $O\left(n+k p^{2}\right)$. Both upper bounds are tight.
\end{abstract}

Mathematics Subject Classifications: 52C45, 52C15

\section{Introduction}

For a finite family $\mathcal{C}=\left\{C_{1}, C_{2}, \ldots, C_{n}\right\}$ of geometric objects in general position in the plane, the union complexity of $\mathcal{U}(\mathcal{C})=\cup_{i=1}^{n} C_{i}$ (or, in short, the union complexity of $\mathcal{C}$ ) is the number of vertices on the boundary $\partial(\mathcal{U}(\mathcal{C}))$, where a vertex is an intersection point of the boundaries of two objects $C_{i}, C_{j} \in \mathcal{C} .^{1}$ More generally, for any $k \geqslant 0$, the $k$-level complexity of $\mathcal{C}$ is the number of vertices that are contained in the interior of exactly $k$ elements of $\mathcal{C}$.

\footnotetext{
*Research partially supported by Grant 635/16 from the Israel Science Foundation, by the Shulamit Aloni Post-Doctoral Fellowship of the Israeli Ministry of Science and Technology, and by the Kreitman Foundation Post-Doctoral Fellowship.

${ }^{\dagger}$ Research partially supported by Grant 635/16 from the Israel Science Foundation.

${ }^{1}$ Formally, the definition of the union complexity is slightly more complex: it is the total number of faces of all dimensions of the arrangement of the boundaries of the objects, which lie on the boundary of the union (see [1]). We use our simpler definition as in our context, both definitions are clearly equivalent up to a constant factor.
} 
Bounding the union complexity of families of geometric objects is useful for analyzing the running time of various algorithms, and has applications to linear programming, robotics, molecular modeling, and many other fields. In particular, Clarkson and Varadarajan [5] showed that if the union complexity of a family $\mathcal{R}$ of $r$ ranges with $V C$ dimension $\delta$ is sufficiently close to $O(r)$, then $\mathcal{R}$ has an $\epsilon$-net of size smaller than $O\left(\frac{\delta}{\epsilon} \log \frac{\delta}{\epsilon}\right)$; Smorodinsky [11] showed that bounds on the union complexity and on the level-1 complexity of families of geometric objects in the plane can be used in computing the proper chromatic number and the conflict-free chromatic number of the corresponding hypergraph. Union complexity of various families has been studied extensively; $k$-level complexity was also the subject of extensive study and is sometimes considered harder to evaluate. For example, finding the $k$-level complexity of a family of $n$ half-planes is the well-known ' $k$-set problem' posed by Lovász (1971) and Erdős et al. (1973), which is still wide open (see, e.g., [6, 12]). For more on union complexity and $k$-level complexity, see the survey [1] and [10, Chapter 11].

For several families of geometric objects, it was shown that the union complexity is asymptotically lower than the trivial $O\left(n^{2}\right)$ bound. In particular, Kedem et al. [9] showed that the union complexity of any family of $n$ pseudo-discs in the plane is at most $6 n-12$, and Alt et al. [2] and Efrat et al. [7] proved a similar bound for any family of fat wedges. An almost linear bound for families of $\gamma$-fat triangles was obtained by Ezra et al. [8].

For a general family of axis-parallel rectangles in the plane, the union complexity can be quadratic - e.g., if the family is an $\frac{n}{2}$-by- $\frac{n}{2}$ grid of long and thin rectangles. However, one may note that such a family contains as many as $n / 2$ pairwise disjoint sets. Hence, it is natural to ask whether any family of axis-parallel rectangles with a quadratic union complexity must contain a linear-sized sub-family whose elements are pairwise disjoint.

In this note we answer this question in the affirmative. We show that the union complexity of any family $\mathcal{R}$ of axis-parallel rectangles is sub-quadratic if the packing number of the family is sub-linear. Recall that the packing number of $\mathcal{R}$, denoted $\nu(\mathcal{R})$, is $p-1$ if $p$ is the smallest number such that among any $p$ elements of $\mathcal{R}$, two have a non-empty intersection. Our main result is the following:

Theorem 1. Let $\mathcal{R}$ be a family of axis-parallel rectangles in general position with packing number $\nu(\mathcal{R})$. Then for any $k \geqslant 1$, the $(k-1)$-level complexity of $\mathcal{R}$ is $O\left(n+k \nu(\mathcal{R})^{2}\right)$. In particular, the union complexity of $\mathcal{R}$ is $O\left(n+\nu(\mathcal{R})^{2}\right)$.

Both results are tight, as we show by an explicit example. ${ }^{2}$

\section{Proof of Theorem 1}

The proof of Theorem 1 consists of several steps, and for convenience we divide them into separate subsections. We start with a few definitions and notations.

\footnotetext{
${ }^{2}$ We note that our upper bound on the union complexity is not hereditary, in the sense that there may exist a sub-family of $\mathcal{R}$ (of size $\Theta(p)$, where $\nu(\mathcal{R})=p-1$ is the packing number of $\mathcal{R}$ ) whose union complexity is quadratic in its number of elements. Another non-hereditary bound on the union complexity, for specific families of discs in the plane, was obtained by Aronov et al. [3].
} 


\subsection{Definitions and Notations}

Throughout this note, $\mathcal{R}$ denotes a family of axis-parallel rectangles in the plane, and we assume that $\mathcal{R}$ is in general position, meaning that no two rectangles have more than 4 common points (i.e., no two rectangles share a segment of the boundary; this implies that no three boundaries intersect at the same point). Put $\nu(\mathcal{R})=p-1$, so any $p$ rectangles in $\mathcal{R}$ contain two with a non-empty intersection.

For any $x \in \mathbb{R}^{2}$, the depth of $x$, denoted depth $(x)$, is the number of rectangles in $\mathcal{R}$ that contain $x$ as an interior point. For $k \geqslant 0$, let $Y_{k}$ be the set of vertices (i.e., intersections of pairs of boundaries) of depth $k$. Of course, $\left|Y_{0}\right|$ is the union complexity of $\mathcal{R}$ and $\left|Y_{k}\right|$ is the $k$-level complexity of $\mathcal{R}$.

\subsection{Partition of the rectangles into floors}

Let $R_{1} \in \mathcal{R}$ be the rectangle whose upper boundary is the lowest (i.e., has the smallest $y$ coordinate) among the rectangles in $\mathcal{R}$. If there are several such rectangles, we choose one of them arbitrarily. Denote by $\ell_{1}$ the horizontal line that contains the upper boundary of $R_{1}$.

Define inductively a sequence $\left\{\ell_{i}\right\}_{2 \leqslant i \leqslant p^{\prime}}$, for some $p^{\prime} \leqslant p$, as follows. Let $R_{i}$ be the rectangle whose upper boundary is the lowest between all elements of $\mathcal{R}$ whose lower boundary is above $\ell_{i-1}$. (Again, if there are several such rectangles, we pick one of them arbitrarily.) Denote by $\ell_{i}$ the horizontal line that contains the upper boundary of $R_{i}$. If there are no rectangles in $\mathcal{R}$ whose lower boundary is above $\ell_{i-1}$, take $\ell_{i}$ to be an arbitrary horizontal line above $\ell_{i-1}$, set $p^{\prime}=i$, and stop the process. Note that by the construction, the rectangles $\left\{R_{i}\right\}_{1 \leqslant i \leqslant p^{\prime}}$ are pairwise disjoint. As $\nu(\mathcal{R})=p-1$, this implies that the process in indeed finite and that $p^{\prime} \leqslant p$.

We now define the partition of $\mathcal{R}$ into floors: we say that $R \in \mathcal{R}$ belongs to floor $i$, $1 \leqslant i \leqslant p^{\prime}-1$, if the upper boundary of $R$ is above or contained in $\ell_{i}$ and lower than $\ell_{i+1}$. We denote the set of all rectangles in floor $i\left(1 \leqslant i \leqslant p^{\prime}-1\right)$ by $\mathcal{F}_{i}$. It is clear from the construction that $\left\{\mathcal{F}_{i}\right\}_{1 \leqslant i \leqslant p^{\prime}-1}$ is a partition of $\mathcal{R}$ into $p^{\prime}-1 \leqslant p-1$ pairwise disjoint families. In addition, we need the following observation:

Observation 2. For any $1 \leqslant i \leqslant p^{\prime}-1$, if $R \in \mathcal{F}_{i}$ then $R \cap \ell_{i} \neq \emptyset$. Furthermore, $i$ is the largest index such that $R$ intersects $\ell_{i}$.

Proof. Let $R \in \mathcal{F}_{i}$. If the lower boundary of $R$ is above $\ell_{i}$ then by the definition of $\ell_{i+1}$, the upper boundary of $R$ cannot lie strictly below $\ell_{i+1}$, a contradiction. Hence, the lower boundary of $R$ is either below $\ell_{i}$ or on $\ell_{i}$. As the upper boundary of $R$ is either on $\ell_{i}$ or above $\ell_{i}$ and also lower than $\ell_{i+1}$, the assertion follows.

Observation 2 implies that $\mathcal{R}$ is pierced by the set of lines $\mathcal{L}=\left\{\ell_{1}, \ldots, \ell_{p^{\prime}-1}\right\}$, meaning that each $R \in \mathcal{R}$ has a non-empty intersection with (at least) one of the lines. A similar argument shows that there exists a set $\mathcal{H}=\left\{h_{1}, h_{2}, \ldots, h_{p^{\prime \prime}-1}\right\}$ (for some $p^{\prime \prime} \leqslant p$ ) of vertical lines (arranged in increasing order of the $x$ coordinate) that pierces $\mathcal{R}$. We may assume w.l.o.g. that $p^{\prime \prime}=p$. The set $\mathcal{H}$ will be used, along with $\mathcal{L}$, in the sequel. 


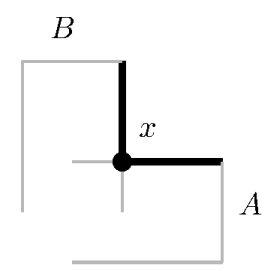

(a)

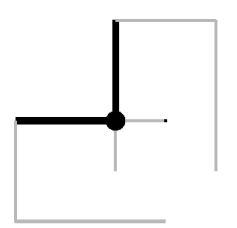

(b)

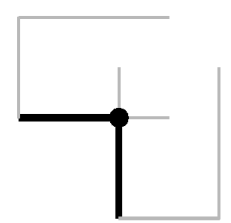

(c)

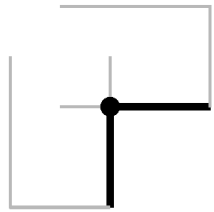

(d)

Figure 1: Types of intersection of pairs of axis-parallel rectangles in general position.

\subsection{Classification of the intersection points of type $L$}

Intersection points of boundaries of two axis-parallel rectangles can be partitioned into four types, depicted in Figure 1. The type described in Figure 1(a) (in which the intersection point is the rightmost-upmost point of the intersection of the rectangles) will be called type $L$ intersection. We denote by $X_{k}$ the set of all points of type $L$ in $Y_{k}$. In what follows, we obtain an upper bound on $\left|X_{k}\right|$. By symmetry, this will imply an upper bound on the $k$-level complexity of $\mathcal{R}$. As a preparation, we classify the intersection points of type $L$.

For any intersection point $x$ of type $L$, we denote by $A_{x}$ the rectangle to whose upper boundary $x$ belongs, and by $B_{x}$ the rectangle to whose right boundary $x$ belongs.

Definition 3. Let $x \in X_{k}$. Denote by $h_{x}$ the rightmost amongst the vertical lines in the set $\left\{h \in \mathcal{H}: h \cap B_{x} \neq \emptyset\right\}$. We say that $x$ is $\left(A_{x}, h_{x}\right)$-contributed.

For $A \in \mathcal{R}$, we say that $x$ is $A$-contributed if there exists $h \in \mathcal{H}$ such that $x$ is $(A, h)$ contributed. Conversely, for $h \in \mathcal{H}$, we say that $x$ is $h$-contributed if there exists $A \in \mathcal{R}$ such that $x$ is $(A, h)$-contributed (see Figure 2(a)).

Observation 4. 1. For any given $A, h, k$, there exists at most a single point $x$ with $\operatorname{depth}(x)=k$ that is $(A, h)$-contributed.

2. It may be that $x$ is $(A, h)$-contributed, while $A \cap h=\emptyset$ (see Figure $2(b)$ ).

Definition 5. An $(A, h)$-contributed point $x$ is called an inner contribution of $A$ if there exist points $y, z$ and lines $h \neq h^{\prime}, h^{\prime \prime} \in \mathcal{H}$, such that:

- $y$ is $\left(A, h^{\prime}\right)$-contributed and $z$ is $\left(A, h^{\prime \prime}\right)$-contributed, and

- $x$ lies strictly between $y$ and $z$. (Note that all of $x, y, z$ belong to the upper boundary of $A$. This induces a natural ordering between them.)

If there are no such points, $x$ is called an extremal contribution of $A$ (see Figure 2(c)).

The following observation is crucial in the sequel.

Observation 6. Let $x \in X_{k}$ be an $\left(A, h_{i}\right)$-contributed intersection point. If $x$ is an inner contribution of $A$, then $A$ intersects both $h_{i}$ and $h_{i+1}$. 


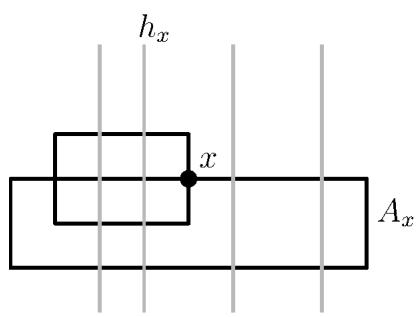

(a)

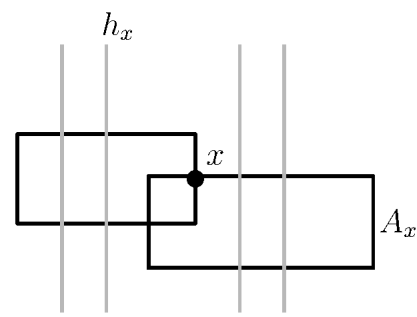

(b)

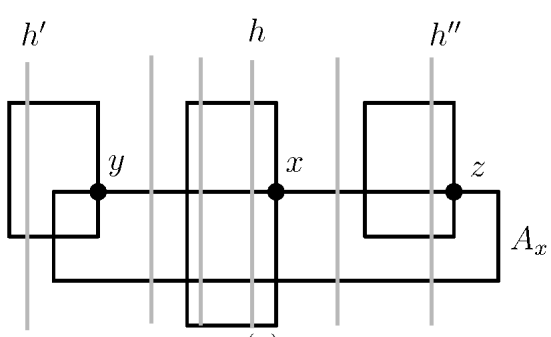

$(c)$

Figure 2: An auxiliary figure for Section 2.3. In (a) and (b), the point $x$ is $\left(A_{x}, h_{x}\right)$ contributed. In (c), $x \in X_{0}$ is an inner contribution of $A$.

Proof. Denote the vertical lines that contain the left and right boundaries of $A$ by $l_{A}$ and $r_{A}$, respectively. Note that if for some $m$ there exists an $\left(A, h_{m}\right)$-contributed point $\bar{x}$, then the line $h_{m+1}$ must lie to the right of $l_{A}$ (as otherwise, $B_{\bar{x}}$ must intersect $h_{m+1}$, contradicting the assumption that $\bar{x}$ is contributed by $h_{m}$ ). On the other hand, $h_{m}$ must lie to the left of $r_{A}$, since it intersects $B_{\bar{x}}$ and the right boundary of $B_{\bar{x}}$ is to the left of $r_{A}$ (as the intersection point $\bar{x}$ is of type $L$, see Figure 1(a)).

In our case, as $x$ is an inner contribution of $A$, there exist some $s_{1}, s_{2} \geqslant 1$ and points $y, z$ such that $y$ is $\left(A, h_{i-s_{1}}\right)$-contributed and $z$ is $\left(A, h_{i+s_{2}}\right)$-contributed. By the previous paragraph, the former implies that $h_{i-s_{1}+1}$ lies to the right of $l_{A}$ while $h_{i+s_{2}}$ lies to the left of $r_{A}$. As $s_{1}, s_{2} \geqslant 1$, this implies that both $h_{i}$ and $h_{i+1}$ lie to the right of $l_{A}$ and to the left of $r_{A}$, and thus, both intersect $A$, as asserted.

\subsection{Upper bound on 'inner contributions' to the k-level complexity}

In this subsection we obtain an upper bound on the number of elements of $X_{k}$ that are inner contributions, by considering pairs of the form (Floor $\mathcal{F}_{i}$, vertical line $h_{j}$ ) separately, and for each such pair, upper bounding the number of $\left(A, h_{j}\right)$-contributed points for $A \in \mathcal{F}_{i}$ that are inner contributions.

Proposition 7. For $k \geqslant 0,1 \leqslant i \leqslant p^{\prime}-1$, and $1 \leqslant j \leqslant p-1$, let

$S_{k}^{i, j}=\left\{x \in X_{k}: \exists A \in \mathcal{F}_{i}, x\right.$ is $\left(A, h_{j}\right)$-contributed and $x$ is an inner contribution of $\left.A\right\}$.

(Informally, $S_{k}^{i, j}$ is the set of all contributions to the $k$-level complexity, that are contributed by $h_{j}$ on the $i$-th floor in an 'inner' way). Then for all $i, j$,

$$
\left|S_{k}^{i, j}\right| \leqslant k+1 \text {. }
$$

Proof. Fix $1 \leqslant j \leqslant p-1$. Define, for any $1 \leqslant i \leqslant p^{\prime}-1$,

$$
\mathcal{A}_{i}=\left\{A \in \mathcal{F}_{i}: \exists\left(A, h_{j}\right) \text {-contributed } x \in X_{k} \text { that is an inner contribution of } A\right\} .
$$

(Informally, $\mathcal{A}_{i}$ is the set of all rectangles on the $i$-th floor, whose upper edge contains an inner contribution to the $k$-level complexity, contributed by $h_{j}$.) Denote $\left|\mathcal{A}_{i}\right|=m_{i}$, and 


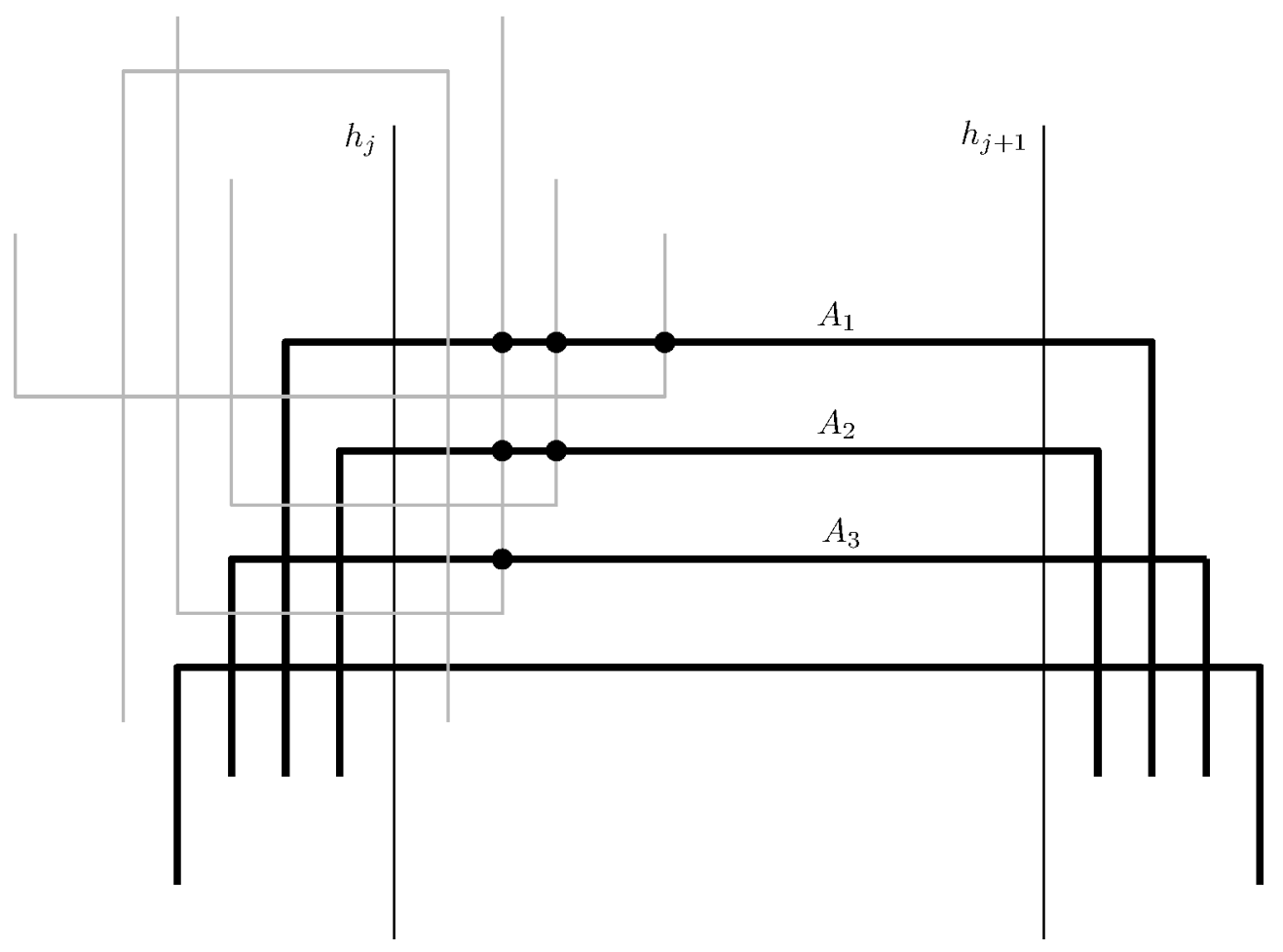

Figure 3: An illustration to the proof of Proposition 7.

let the elements of $\mathcal{A}_{i}=\left\{A_{1}, A_{2}, \ldots, A_{m_{i}}\right\}$ be ordered in descending order of the height of the upper boundary, as demonstrated in Figure 3. (So, $A_{1}$ is the rectangle whose upper boundary is the highest, $A_{2}$ 's upper boundary is the second highest, etc.. Note that equality cannot occur here as by Observation 6, any $A \in \mathcal{A}_{i}$ intersects both $h_{j}$ and $h_{j+1}$, and so, if two of these rectangles had upper boundaries of the same height, they would share part of the boundary, contradicting the assumption that the elements of $\mathcal{R}$ are in general position.)

For each $1 \leqslant l \leqslant m_{i}$, denote

$$
Q_{l}=\left\{x \in X_{k}: x \text { is }\left(A_{l}, h_{j}\right) \text {-inner contributed }\right\} .
$$

By Observation 4, for each $l$ we have $\left|Q_{l}\right| \leqslant 1$. It is clear that

$$
\left|S_{k}^{i, j}\right|=\mid\left\{x \in X_{k}: \exists A \in \mathcal{A}_{i} \text { such that } x \text { is }\left(A, h_{j}\right) \text {-inner contributed }\right\}\left|=\sum_{l=1}^{m_{i}}\right| Q_{l} \mid .
$$

Hence, the assertion will follow once we show that $Q_{l}=\emptyset$ for all $l>k+1$. To see this, we prove that for each $l$, each $x \in Q_{l}$, and each $1 \leqslant r \leqslant l-1, x$ is an interior point of $A_{r}$ (and thus, each $x \in Q_{l}$ is of depth $\geqslant l-1$ ). We use several simple observations.

1. Any $\left(A_{l}, h_{j}\right)$-contributed $x$ lies between the lines $h_{j}$ (inclusive) and $h_{j+1}$ (noninclusive). Indeed, as $x$ lies on the right boundary of $B_{x}$ and $h_{j}$ intersects $B_{x}$, 
$x$ must lie either on $h_{j}$ or to the right of $h_{j}$. On the other hand, if $x$ lies on $h_{j+1}$ or on the right of $h_{j+1}$, then $B_{x}$ must intersect $h_{j+1}$, a contradiction.

2. Any such $x$ lies above or on the line $\ell_{i}$, since it belongs to the upper boundary of $A_{l} \in \mathcal{F}_{i}$.

3. Each of the rectangles $A_{1}, \ldots, A_{m_{i}}$ intersects $\ell_{i}$ by Observation 2 , and intersects both $h_{j}$ and $h_{j+1}$ by Observation 6 .

By the simple observations, for each $1 \leqslant r \leqslant l-1$, the rectangle $A_{r}$ intersects $\ell_{i}, h_{j}$ and $h_{j+1}$, and its upper boundary lies above $x$ (since $x$ lies on the upper boundary of $A_{l}$ ). As $x$ lies between the lines $h_{j}$ and $h_{j+1}$ and above $\ell_{i}$, it follows that $x$ is an interior point of $A_{r}$. This completes the proof.

\subsection{Finalizing the proof of Theorem 1}

Now we are ready to prove Theorem 1. Actually, we prove the following exact version of the theorem:

Theorem 8. Let $\mathcal{R}$ be a family of $n$ axis-parallel rectangles in general position with $\nu(\mathcal{R})=p-1$. For any $k \geqslant 0$, the $k$-level complexity of $\mathcal{R}$ is at most $8 n+4(p-1)(p-$ $3)(k+1)$. In particular, the union complexity of $\mathcal{R}$ is at most $8 n+4(p-1)(p-3)$.

Proof. By symmetry considerations, the $k$-level complexity of $\mathcal{R}$ is at most $4\left|X_{k}\right|$, so it is sufficient to prove

$$
\left|X_{k}\right| \leqslant 2 n+(p-1)(p-3)(k+1) .
$$

We prove (3) by upper bounding the inner contributions and the extremal contributions separately.

Inner contributions. By Proposition 7 , for each $i, j$, the number of inner contributions that correspond to $\mathcal{F}_{i}$ and $h_{j}$ is at most $k+1$. For $j \in\{1, p-1\}$, any $h_{j}$-contributed $x$ is an extremal contribution. Hence, the number of inner contributions that correspond to $\mathcal{F}_{i}$ is at most $(p-3)(k+1)$, and so, the total number of inner contributions is at most $(p-1)(p-3)(k+1)$.

Extremal contributions. Let $A \in \mathcal{R}$. By the definition of inner and extremal contributions, all $A$-contributed points that are extremal contributions belong to one of two vertical lines. By Observation 4, for any single pair $(A, h), X_{k}$ contains at most one $(A, h)$-contributed point. Therefore, there are at most two $A$-contributed points that are extremal contributions. It follows that the total number of extremal contributions is at most $2 n$. This completes the proof.

Remark 9. Theorem 8 implies that the $(\leqslant k)$-level complexity of $\mathcal{R}$ is at most $8(k+1) n+$ $2(p-1)(p-3)(k+1)(k+2)$. We note that a similar bound on the $(\leqslant k)$-level complexity can be achieved by first obtaining an upper bound on the union complexity of $\mathcal{R}$ and then applying the classical technique of Clarkson and Shor [4] (which bounds the $(\leqslant k)$-level 

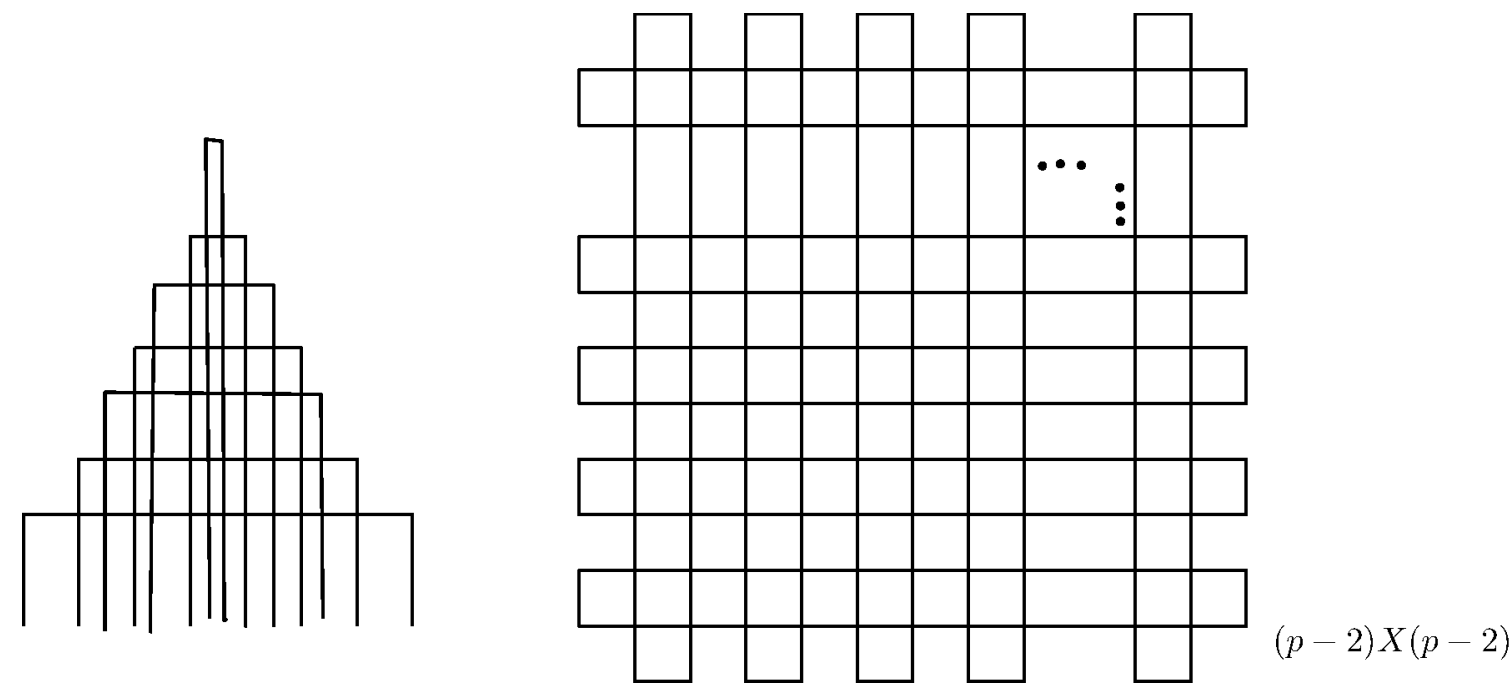

Figure 4: A family of axis-parallel rectangles that demonstrates the tightness of Theorem 1.

complexity of a family $\mathcal{R}^{\prime}$ using a bound on the union complexity of a random sub-family in which each element of $\mathcal{R}^{\prime}$ is selected with probability $\left.1 / k\right)$. It seems, however, that the Clarkson-Shor technique does not provide an effective bound on the $k$-level complexity, and thus is not sufficient for proving Theorem 8 .

\section{Tightness of Theorem 1}

In this section we present a family $\mathcal{R}$ of $n$ axis-parallel rectangles with $\nu(\mathcal{R})=p-1$ whose $(k-1)$-level complexity is $\Theta\left(n+k p^{2}\right)$, thus showing that Theorem 1 is tight (up to a constant factor). We note that in our construction, we assume that $k+1 \leqslant \frac{n}{4(p-2)}$.

The family $\mathcal{R}$, presented in Figure 4 , is a disjoint union of two subfamilies of $n / 2$ rectangles each.

The subfamily drawn in the left of the figure consists of a sequence of pairwiseintersecting rectangles in which each rectangle is taller and thinner than its successor. This subfamily contributes $O(n)$ points to the $k$-level complexity of $\mathcal{R}$.

The subfamily drawn in the right of the figure is based on an $(p-2)$-by- $(p-2)$ grid of long thin rectangles. We replace each rectangle in the basic grid with $\frac{n}{4(p-2)}$ nested copies to obtain a family of $n / 2$ rectangles (for simplicity, we assume $4(p-2) \mid n$; note that only the basic grid is depicted in the figure). This subfamily contributes $\Theta\left(k p^{2}\right)$ points to the $k$-level complexity of $\mathcal{R}$. (Here we use the assumption $\frac{n}{4(p-2)} \geqslant k+1$.)

Hence, the $(k-1)$-level complexity of $\mathcal{R}$ is $\Theta\left(n+k p^{2}\right)$, as asserted. 


\section{References}

[1] P. K. Agarwal, J. Pach and M. Sharir, State of the union, of geometric objects: A review, in Proc. Joint Summer Research Conf. on Discrete and Computational Geometry: 20 Years Later, Contemp. Math. 452 (2008), AMS, pp. 9-48.

[2] H. Alt, R. Fleischer, M. Kaufmann, K. Mehlhorn, S. Näher, S. Schirra, and C. Uhrig, Approximate motion planning and the complexity of the boundary of the union of simple geometric figures, Algorithmica 8 (1992), pp. 391-406.

[3] B. Aronov, M. Dulieu, R. Pinchasi, and M. Sharir, On the union complexity of diametral disks, Electr. J. Comb. 20(2) (2013), \#P53.

[4] K. L. Clarkson and P. W. Shor, Applications of random sampling in computational geometry, II, Disc. Comput. Geom. 4 (1989), pp. 387-421.

[5] K. L. Clarkson and K. Varadarajan, Improved approximation algorithms for geometric set covers, Disc. Comput. Geom. 37 (2007), pp. 43-58.

[6] T. L. Dey, Improved bounds for planar $k$-sets and related problems, Disc. Comput. Geom. 19(3) (1998), pp. 373-382.

[7] A. Efrat, G. Rote, and M. Sharir. On the union of fat wedges and separating a collection of segments by a line, Comput. Geom. Theory Appl. 3 (1993), pp. 277-288.

[8] E. Ezra, B. Aronov, and M. Sharir, Improved bound for the union of fat triangles, proceedings of SODA'2011, pp. 1778-1785.

[9] K. Kedem, R. Livne, J. Pach, and M. Sharir. On the union of Jordan regions and collision-free translational motion amidst polygonal obstacles in the plane, Disc. Comput. Geom. 1 (1986), pp. 59-71.

[10] J. Matoušek, Lectures on Discrete Geometry, Springer-Verlag New York, 2002.

[11] S. Smorodinsky, On the chromatic number of some geometric hypergraphs, SIAM J. Disc. Math., 21(3) (2007), pp. 676-687.

[12] G. Tóth, Point sets with many k-sets, Disc. Comput. Geom. 26(2) (2001), pp. 187194. 